\title{
Rate of Growth of Bacillus cereus Between Divisions
}

\author{
By J. F. COLLINS AND M. H. RICHMOND \\ Bacterial Physiology Division, National Institute for Medical Research, \\ The Ridgeway, Mill Hill, London, N.W. 7
}

(Received 18 May 1961)

\begin{abstract}
SUMMARY
Bacillus cereus organisms growing exponentially have a stable length distribution. This length distribution can be analysed by the method described to give the mean rate of increase in length of organisms at any given length. The validity of the method was confirmed by observing the growth of clones of $B$. cereus in the culture chamber. Both methods showed that the rate of increase in length increased as the organisms got longer, and that there was no hesitation before or after division. Possible applications of this general method to other parameters that can be measured in samples of bacteria taken from stable populations are suggested.
\end{abstract}

\section{INTRODUCTION}

The growth of bacteria is a co-ordinated process involving the duplication of the various components of the cell in such a way that new viable organisms can be formed with a high probability by any individual organism. Certain aspects of this process have long been a source of study, but no simple model of bacterial growth between divisions has been found which fits the observed behaviour of individual organisms. This is probably explained by the large variations in behaviour of apparently similar organisms growing under similar conditions. Many measurements of the growth of individual organisms between divisions have been made, and a variety of patterns has been observed. For example, steady exponential increase in length was reported by Ward (1895) for Bacillus ramosus and by Adolph \& Bayne-Jones (1932) for B. megaterium. Sigmoid curves were observed by Bayne-Jones \& Adolph (1932a) for the increase in volume of Saccharomyces cerevisiae, while the rate of increase in length decreased throughout the growth cycle; Knaysi obtained similar results for the increase in length of Schizosaccharomyces pombe (1940) and the increase in volume of Streptococcus faecalis (1941). BayneJones \& Adolph (1932b) found a variety of curves of increasing length and volume in a single experiment with Escherichia coli. Steady, accelerating increase in length between divisions was suggested by Knaysi (1940) for B. cereus. The methods used by all these authors entailed observations on single organisms growing on the surface of some solid medium or in a hanging-drop preparation, and in no case has it been possible to compare the results with the behaviour of the same bacteria growing under more usual conditions in bulk liquid culture. Powell $(1955,1958)$ made large numbers of observations of the generation times of individual bacteria growing in a special chamber (Powell, 1956) in which the growth conditions were closer to bulk liquid conditions, but he did not investigate the rate of increase in length between divisions. When the growth of single clones is studied in a culture chamber of the 
type mentioned, it is possible to obtain the average rate of increase in length between divisions directly; but this requires prolonged observations under carefully controlled conditions to obtain a statistically significant sample.

The object of the work to be described was to measure the rate of increase in length of Bacillus cereus between divisions from an analysis of the relative distribution of lengths of organisms in an exponentially growing culture and thus to avoid the limitations implicit in the study of single organisms. The method which has been developed to calculate the rate of increase in length from the length distribution of the organisms in such a sample depends on certain characteristics of an exponentially growing culture, which are not necessarily true of cultures in other phases of growth. B. cereus organisms approximately double in length between divisions, and in a growing posulation organisms are found at all stages of the growth cycle. If the age of an o ganism is regarded as the time since it was formed by division, then in an exponentially growing culture there are always more younger organisms than older organisms, and the distribution of length in a culture of this type usually shows a large propertion of short organisms (see Henrici, 1928). There is no unique length at which the organisms divide and division is observed to occur over a range of lengths among the larger organisms. The new organisms formed by division thus also appear at various lengths.

In a truly exponential growing vulture, the distribution of lengths of the organisms can be shown to be constant, although this state may not be achieved until several generations after the growth ha: apparently become exponential as measured by turbidity or viable count. This distribution can then be regarded as characteristic of the stable population. If this distribution is to remain unchanged as the culture grows further, the proportion of the population with a given length, $x$, must also be constant. The number of organisms of length $x$ must increase exponentially with the same exponential growth coastant as the culture itself. In any time $t$, the increase in numbers of organisms (if length $x$ can be calculated from the exponential growth equation, and this increase in numbers must be the difference between the number of organisms reaching length $x$ in time $t$ and the number of organisms leaving the length $x$ in time $t$. The number of organisms reaching length $x$ in time $t$ has two components; namely, the number of organisms smaller than $x$ growing to length $x$ in time $t$ (component no. 1) and the number of organisms of length $x$ which are formed by the division of larger organisms (not necessarily of length $2 x$, since division is not always symmetrical) in the time $t$ (component no. 2). Similarly, the number of organisms leaving length $x$ in time $t$ is composed of the number of organisms of length $x$ that grow larger than $x$ in time $t$ (component no. 3) plus the number of organisms of length $x$ that divide in time $t$ (component no. 4). 'Thus, the rate of increase in numbers of organisms of any particular length which is necessary to maintain the length distribution of the population constant during exponential growth may be resolved into four components; a knowledge of the stable length distribution and three of these components will allow the remaining component to be deduced. It is component no. 3 the number of organisms of length $x$ that grow larger than $x$ in time $t$ ) that is directly related to the mean rate of increase in length of organisms of this length. Components 1, 2 and 4 are independent of this rate of increase in length and may be estimated separately to allow component no. 3 to be deduced and ultimately the mean rate of increase in length of organisms 
of length $x$ to be calculated. Component no. 1, the number of organisms smaller than $x$ growing to length $x$ in time $t$, is clearly related to the number of organisms that are just shorter than $x$ and their mean rate of increase in length. However, component no. 1 can be more profitably regarded as the number of organisms smaller than $x$ that, taking due regard of the lengths of organisms dividing and being formed in time $t$, must be displaced from the portion of the total culture that is smaller than $x$ in order to maintain a constant proportion of the population in this group as the culture grows. The frequency distributions of length of the dividing and newly formed organisms must now be known in order to evaluate component no. 1; however, this is not simply changing one unknown for another, for these distributions are also required to enable components nos. 2 and 4 to be calculated. Hence, from the stable length distribution of the population and the two frequency distributions of length of the dividing and newly formed organisms, it is possible to determine the mean rate of increase in length of organisms of any length. The precise manner by which this may be accomplished is described in the Appendix.

The two frequency distributions necessary for the calculation in addition to the stable length distribution express the probability of any division or appearance occurring at any given length. This information cannot be deduced directly from fixed samples of organisms taken from a bulk culture. It is, however, possible to give approximate values to these distributions (which will be referred to as the length distributions of dividing and newly formed organisms) which are consistent with the overall length distribution. While the distribution of dividing and newly formed organisms may be of many different statistical types, it was found that the normal distributions with different parameters tested produced only minor differences in the calculated rate of increase in length between divisions. From each normal distribution chosen it was possible to calculate a rate of increase in length of organisms in each length category in the length distribution of the population, and from these results to obtain a general description of the behaviour of organisms over the whole growth cycle between divisions. Each distribution tested produced an identical description, except close to the two extremes of the length distribution, where the rates of division and formation become increasingly significant in the calculation. Changes of variance (at constant mean length) and changes of mean length (with constant variance) in the distributions used produced no significant changes in the calculated values of the rate of increase in length over the major portion of the length distribution. Based on the identical results obtained from these assumed distributions, the rate of increase in length between divisions of Bacillus cereus growing exponentially in casein hydrolysate medium was found to be of a continuously accelerating type. Since these results are of a statistical nature, it is possible that a certain average rate of increase in length may result from a population which is composed of individual organisms, some of which are growing faster than average, and others slower than average, or which have even stopped growing completely. Only observations of individual organisms during the growth cycle can provide a complete description of the individual variation in behaviour which does occur; however, the results obtained by the method described in this paper stand in their own right as a valid description of the average behaviour of the organisms of a bulk culture, from which individual organisms cannot be taken without altering in some degree the conditions under which they have been growing. 
This problem of growth between divisions has also been approached by Scherbaum (1956; Scherbaum \& Rasch, 1957), who gave a limited treatment for the growth of a protozoon (Tetrahymena pyriformis).

In order to check the assumptions outlined above and to obtain more accurate information about the rate of increase in length of very short and very long organisms, the growth of several clones was followed in a culture chamber similar to that used by Powell (1956). By observations of this kind it was possible to compare the behaviour of bacterial cultures in the chamber with that of the bulk culture and to check that the assumptions made in the calculations were representative of the growing culture. A good measure of agreement was, in fact, found, and examination of samples taken from a bulk culture may allow a valid description of the rate of increase in length of organisms during growth between divisions, particularly where measurements on single organisms are impracticable. Moreover, this method of determining the rate of increase in length of organisms between divisions is quite general in application, and can equally well be used to interpret the distribution of other components which can be measured during exponential growth. In order to allow the basic method to be followed more easily, the theoretical treatment has been placed in an appendix.

\section{METHODS}

Organisms, media, etc. Bacillus cereus 569/H (Kogut, Pollock \& Tridgell, 1956) was maintained as a spore suspension in distilled water. Organisms for experimental purposes were prepared by inoculating spores $\left(10^{5} / \mathrm{ml}\right.$.) into casein hydrolysate medium (CH) (Kogut et al. 1956) and incubating overnight at $35^{\circ}$. In the morning, a portion of the culture was diluted with ten volumes of fresh medium and shaken in a flask (at least five times the volume of the culture) at $\mathbf{3 5}^{\circ}$ on a graded shaker (Kantorowicz, 1951) at $86 \mathrm{cyc} . / \mathrm{min}$. Growth was followed turbidimetrically.

Measurement of the length distribution of the organisms. Measurements of bacterial length were made on organisms growing in bulk culture and in a culture chamber. The lengths of the organisms were obtained in the following manner.

From the bulk culture. Samples containing about $0.1 \mathrm{ml}$. were withdrawn from the culture and transferred to microscope slides. The bacteria were dried at room temperature and then fixed at $60^{\circ}$ for $3 \mathrm{~min}$. At the end of the experiment all the fixed preparations were stained with $0.5 \%(\mathrm{w} / \mathrm{v})$ crystal violet for $5 \mathrm{~min}$. and washed under standard conditions in $95 \%(\mathrm{w} / \mathrm{v})$ ethanol in water to remove excess stain. The fixed and stained preparations were then dried in air at room temperature for at least $2 \mathrm{hr}$. Black and white photomicrographs of the preparations were made at a magnification of $\times 290$ and the negatives mounted for projection. The slides were projected on to a screen and the length of all the bacteria on each slide measured with a ruler and a pair of dividers. The measurement of the lengths of the organisms was necessarily rather subjective. In single organisms without any sign of a division furrow, the length was measured as the distance between the visible extremities of the organism. In organisms showing a division furrow the organism was counted as a single entity unless a clear division could be seen between the two daughters such that the length of each daughter could be measured separately. The images of the bacteria (when projected) were between 1.5 and $6.0 \mathrm{~cm}$. long (i.e. an overall magnification of about $\times 10,000)$. 
From the culture chamber. A small sample (about $10^{3}$ bacteria) was transferred from a bulk culture growing exponentially to a culture chamber similar to that designed by Powell (1956). The bacteria, growing on the same medium and at the same temperature as the bulk culture, were observed through a microscope using a long distance phase contrast objective and photomicrographs taken at $4 \mathrm{~min}$. intervals for $90 \mathrm{~min}$. The photographic negatives obtained were projected and the lengths of the bacteria measured by the method described above for the fixed and stained organisms.

\section{RESULTS}

Experiments with bulk cultures

The theoretical treatment set out in the Appendix shows that the rate of increase in length of bacteria between divisions can be deduced from the length distribution of a bacterial population and the length distributions of the dividing and newly formed bacteria. For valid results, the bacterial population must be in stable exponential growth, during which the length distribution should be constant. Four samples were therefore taken from a culture which had been growing exponentially for several generations in $\mathrm{CH}$ medium. To avoid misleading results due to sampling a culture at the same stage of any periodic change, the samples $(0 \cdot 1 \mathrm{ml}$.) were taken at intervals unrelated to the doubling time over a period of $1 \mathrm{hr}$., and were then fixed and stained. The length distribution of the bacteria was determined from photomicrographs in the manner described in the Methods section, several frames being counted to give a statistically significant sample. All the bacteria in any frame were measured to avoid any possible discrimination due to unconscious selection by the measurer. The length distributions were markedly skew, but it was shown that the logarithm of the length was normally distributed. Table 1 shows the main characteristics of the distributions of the four samples and the percentages of the log (length) distributions falling outside stated confidence limits, which show good agreement. The means are similar, and Bartlett's test of homogeneity was performed on the variances giving $\chi^{2}$ ( 3 degrees of freedom) $=5.558$ and $P>0 \cdot 1$. Hence the variances are homogeneous. This means that the samples are from a culture with a stable length distribution, and they may be treated as a single distribution for further analysis. Since the length categories in the distribution differ by small increments close to the resolving power of the light microscope, individual organisms may be readily misplaced in the distribution by one unit of length. The overall length distribution was therefore treated by the procedure of Whittaker which retains the zero, first and second moments of the observed distribution while giving the most probable value at each length category (Whittaker \& Robinson, 1948). The original and resulting distributions are shown in Fig. 1.

In order to calculate the rate of increase in length between divisions from the corrected distribution, the distributions of both the dividing and newly formed organisms must be known. In the absence of any direct experimental evidence, estimates of these two distributions were made from the following considerations. The distributions of the dividing and newly formed organisms are not independent; for example, the mean length of organism at the moment of division is twice that of the daughters at the (same) moment they are formed. It is difficult to define the moment of division in a manner capable of experimental test; the process of division 
Table 1. Characteristics of the observed skew distribution of organism length and the normal distribution of log (organism length) in successive samples taken from a bulk culture growing exponentially

Central measures of the Parameters of the distriobserved distribution

$\begin{array}{cccc}\begin{array}{c}\text { Total } \\ \text { no. of } \\ \text { Sample } \\ \text { organisms } \\ \text { in sample }\end{array} & \begin{array}{c}\text { Mean } \\ (\mu)\end{array} & \begin{array}{c}(a) \\ \text { Median } \\ (\mu)\end{array} \\ 1 & 362 & 3 \cdot 27 & 3 \cdot 15 \\ 2 & 426 & 3 \cdot 16 & 3 \cdot 06 \\ 3 & 435 & 3 \cdot 36 & 3 \cdot 22 \\ 4 & 709 & 3 \cdot 31 & 3 \cdot 13\end{array}$

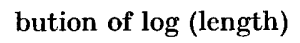

$\begin{array}{lr} & \begin{array}{c}\text { Variance } \\ \times \\ \text { Mean }\end{array} \\ 0.50 & 10^{3} \\ 0.48 & 8 \cdot 71 \\ 0.51 & 9 \cdot 76 \\ 0.50 & 9 \cdot 18 \\ & 10.97\end{array}$

Percentage of log (length) distribution outside stated confidence limits

For purposes of comparison each observed distribution was converted to a normal distribution by plotting the frequency against the logarithm of the observed length.

in bacteria may be observed to occur in several stages during a considerable period of the growth cycle, and it may be argued that it is not possible to speak of the moment of division with any meaning. However, for the treatment used in this paper, it is only necessary to identify a single step of the complete division process as the moment of division, and the choice is arbitrary. As the observations were made with the microscope, the point of the division cycle which was chosen was that at which the two daughter organisms appeared to have a distinct partition between them.

The distributions of the dividing and newly formed organisms are related in variance as well as in mean. If the distributions are normal, two extreme cases can be considered. First, if the organisms split exactly in half, it is possible to reconstruct the length distribution of the parent organisms from the distribution of the newly formed organisms by combining orily pairs of organisms of equal length. The variance of the distribution of the newly formed organisms will be one quarter the variance of the distribution of the dividing organisms. Secondly, if the organisms do not necessarily split in half, and there are no restrictions regarding the sizes of the two pieces that may be recombined in this manner, then the variance of the distribution of the newly formed organisms will be one half the variance of the distribution of the dividing organisms. The real situation lies between these two extreme cases, but it is not possible to deduce it directly from the fixed and stained samples. The distributions of the newly formed and dividing organisms used to calculate the rate of increase in length between divisions are for simplicity assumed to be normal, and must be chosen with variances whose ratio lies within the limits of the two extreme cases described.

From the length distribution of the culture (Fig. $1 b$ ), the following assumptions may be made to aid the choice of suitable values for the means and variances of the distributions of the dividing and newly formed organisms. The smallest organism observed must have been formed at that length or shorter. Since this was the shortest organism observed, the chance that it was formed at a shorter length must be small. The distribution of newly formed organisms must therefore extend to the length of the shortest organism observed (A, Fig. $1 b$ ) but not appreciably beyond. It follows that a limit can also be set to the smallest dividing organism which must be at least 
twice the length of the smallest organism observed (2A, Fig. $1 b)$. The upper size limit observed (B, Fig. $1 b$ ) indicates that organisms of this length must have a high probability of dividing before growing any larger. In addition, there must be a small but finite probability that organisms are formed at lengths greater than half the length of the largest organism observed (B/2, Fig. $1 b)$ because of the possible asymmetric division of such an organism. (It is fortuitous that $2 \mathrm{~A}$ and $\mathrm{B} / 2$ coincide in Fig. $1 b$.) The mean length of the newly formed organisms must lie between $\mathrm{A}$ and $\mathrm{B} / 2$, and since these limits are chosen on similar grounds it is reasonable to assume initially that the mean should lie approximately midway between them. Equally, the mean length of the dividing organisms may be assumed to lie approximately midway between $2 \mathrm{~A}$ and $\mathrm{B}$. Since the mean length of the

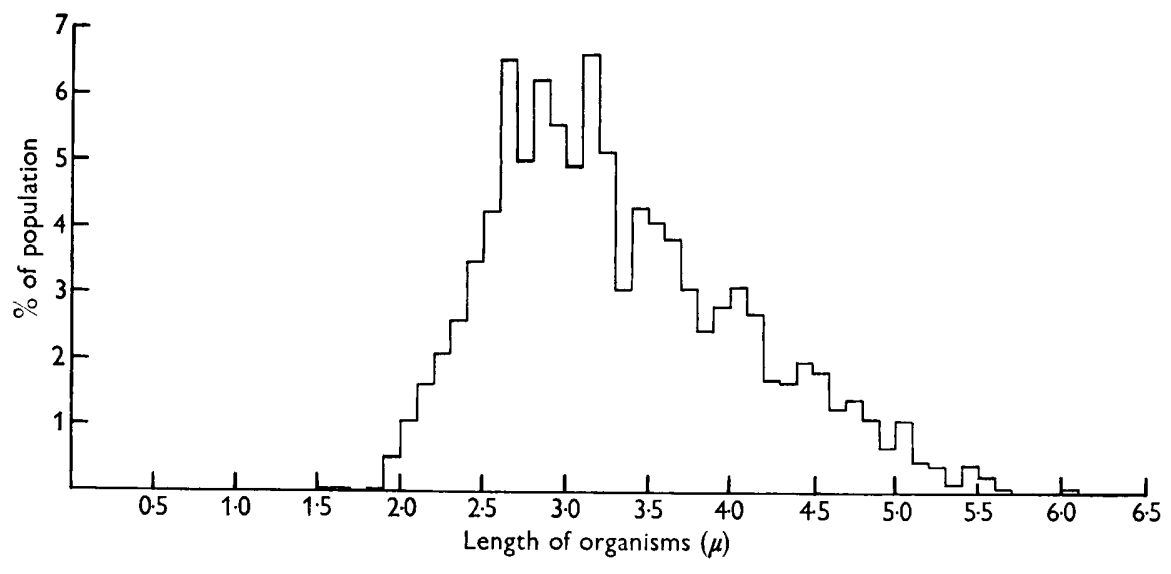

$a$

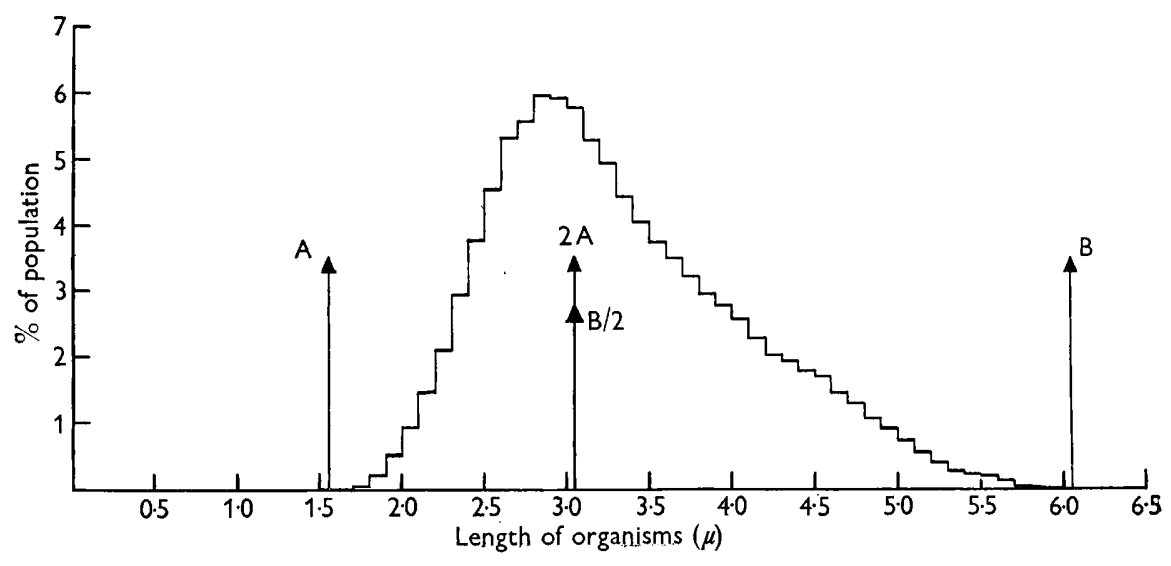

$b$

Fig. 1. Length distribution of organisms in a sample taken from an exponentially growing culture. The total population is made up by pooling the samples taken at intervals during the growth of the bulk culture (see Table 1). The distributions are shown before $(a)$ and after $(b)$ treatment by the method of Whittaker (Whittaker \& Robinson, 1948). $1 b$ also shows the limits used in estimating the means and variances of the length distributions of dividing and newly formed organisms. A: Shortest organism observed; 2A: twice the length of the shortest organism; B: longest observed organism; B/2: half the length of the longest observed organism. 


\section{Table 2. Properties of the distributions of length of dividing and newly formed organisms}

Chosen to study the effect of variation of these parameters on the calculated rate of increase in length of organisms between divisions (for details see text), and properties of the distributions observed in the culture chamber experiment, which were also used to calculate the rate of increase in length of organisms between divisions.

\begin{tabular}{|c|c|c|c|c|}
\hline \multirow{2}{*}{$\begin{array}{l}\text { Reference } \\
\text { no. to } \\
\text { estimates } \\
\text { tested }\end{array}$} & \multicolumn{2}{|c|}{ Dividing organisms } & \multicolumn{2}{|c|}{ Newly formed organisms } \\
\hline & $\begin{array}{c}\text { Mean } \\
(\mu)\end{array}$ & Variance & $\begin{array}{c}\text { Mean } \\
(\mu)\end{array}$ & Variance \\
\hline 1 & $4 \cdot 5$ & 0.09 & $2 \cdot 25$ & 0.0315 \\
\hline 2 & $4 \cdot 4$ & $0 \cdot 09$ & $2 \cdot 2$ & $0 \cdot 0315$ \\
\hline 3 & $4 \cdot 6$ & 0.09 & $2 \cdot 3$ & 0.0315 \\
\hline 4 & $4 \cdot 65$ & $0 \cdot 09$ & $\mathbf{2 \cdot 3 2 5}$ & $0 \cdot 0315$ \\
\hline $\mathbf{5}$ & $\mathbf{5} \cdot \mathbf{0}$ & $0 \cdot 09$ & $\mathbf{2 \cdot 5}$ & 0.0315 \\
\hline 6 & $4 \cdot 65$ & 0.078 & $\mathbf{2 \cdot 3 2 5}$ & 0.0285 \\
\hline 7 & 4.65 & $0 \cdot 15$ & $2 \cdot 325$ & 0.0465 \\
\hline 8 & $5 \cdot 0$ & $0 \cdot 15$ & $2 \cdot 5$ & 0.0465 \\
\hline Observed & $4 \cdot 986$ & $0 \cdot 212$ & $2 \cdot 493$ & 0.080 \\
\hline
\end{tabular}

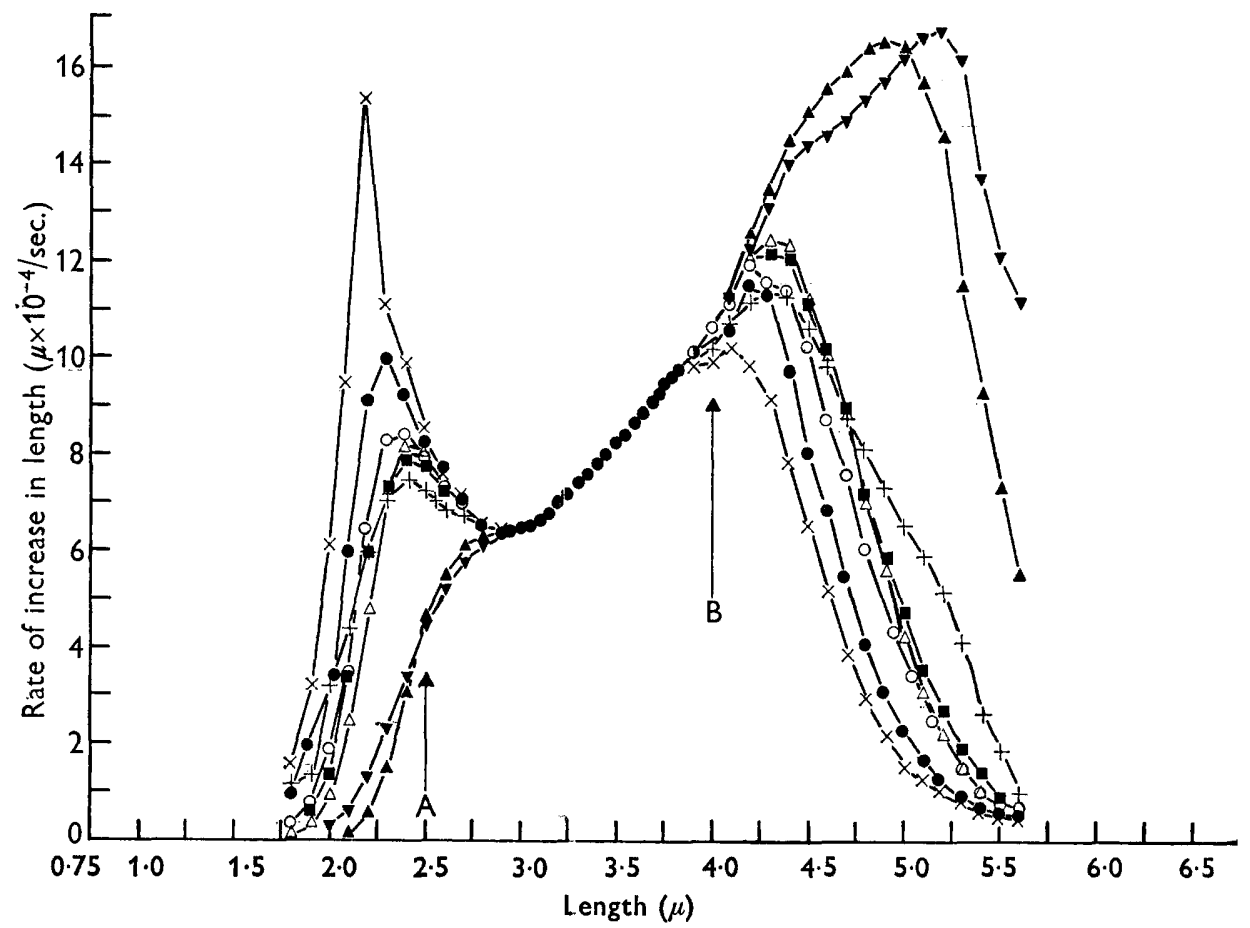

Fig. 2. Rate of increase in length of Bacillus cereus between divisions (plotted against length) calculated from the overall length distribution and the assumed values of the length distributions of the dividing and newly formed organisms. The central portion of the curve lying between $A$ and $B$ is common to all sets of calculated results. The following symbols denote the different distributions given in Table $2:$ no. 1,$0 ;$ no. $2, \times ;$ no. 3,0 ; no. 4 , $\square$; no. $5, \Delta$; no. $6, \Delta$; no. 7 , + ; no. $8, \nabla$. 
dividing organisms must be twice the mean length of the newly formed organisms, and the distributions of dividing and newly formed organisms must extend to cover the limiting cases described above, certain distributions can now be chosen which conform to these requirements for mean and variance, and used in the calculations of the rate of increase in length between divisions. The properties of the normal distributions of length of the dividing and newly formed organisms that have been chosen in this way are shown in Table 2. The rate of increase in length of organisms between divisions was calculated using these distributions, and the results are shown in Fig. 2; to aid interpretation, bacterial length is plotted against time in Fig. 3, and the slope of the curve is the rate of increase in length at any length (from Fig. 2). It is apparent that variations in the mean lengths of the distributions of the dividing and newly formed organisms have also little effect on the shape of the curves except towards the two extremes of the length range. Alterations in the variance of the distributions of the dividing and newly formed organisms have also little effect on the shape of the curve except at the two ends of the length distribution.

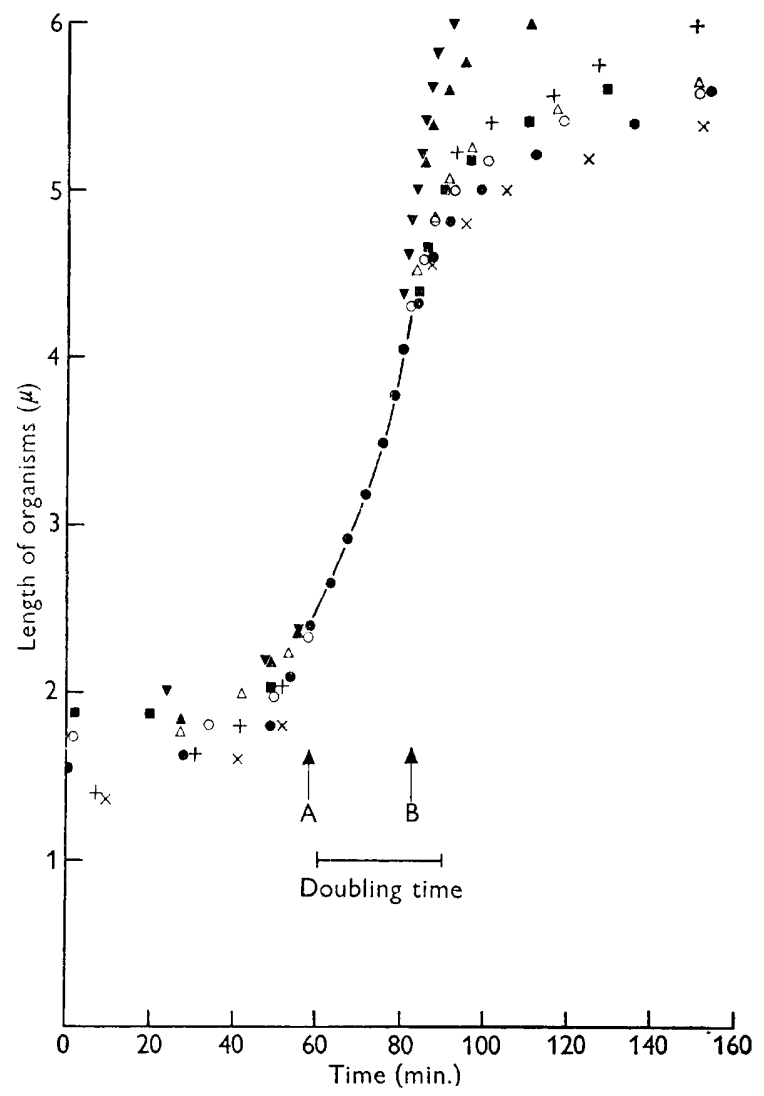

Fig. 3. The rate of increase in length of Bacillus cereus between divisions (plotted as length against time- see text) calculated from the overall length distribution and the assumed values of the length distributions of the dividing and newly formed organisms. The central portion of the curve lying between $A$ and $B$ is common to all length distributions of dividing and newly formed organisms. Outside this range, the following symbols denote the different distributions given in Table 2 ; no. 1,$0 ;$ no. $2, \times$; no. 3,0 ; no. 4, 1 ; no. $5, \Delta$; no. $6, \Delta$; no. 7 , + ; no. $8, \nabla$. Doubling time $=30 \mathrm{~min}$. 

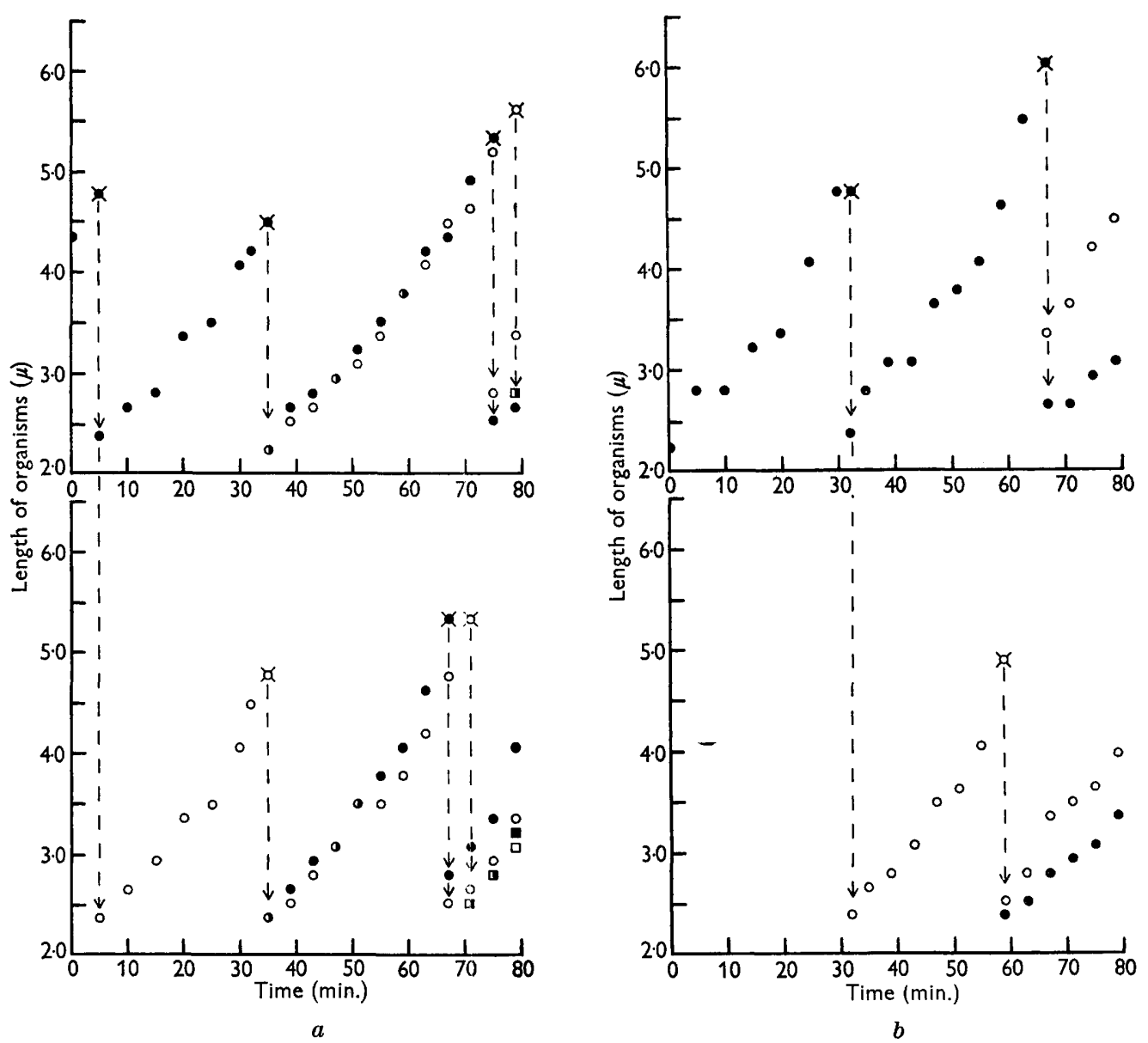

Fig. 4. Growth curves of four clones of Bacillus cereus growing in the culture chamber. After each division the lengths of the daughter organisms are shown by $O$ and 0 . Points having crosses are given by the total length of the two daughter organisms at the first observation after a division has occurred. The dashed lines show the parentage of the organisms. Sister organisms occurring at the same length are shown thus: $\square$ or $\square$.

The rate of increase in length is clearly not constant (i.e. the rate of growth between divisions is not linear) but is continuously accelerating. It does not, however, fit exactly an exponential rate of growth, though the difference is very small; this difference is regarded as real, since the number of organisms analysed is so large.

\section{Experiments on clones growing in the culture chamber}

Since the curves shown in Fig. 3 have been calculated using assumed values for the distributions of the dividing and newly formed organisms, it was decided to test the validity of the assumptions by following the rate of increase in length between divisions of a number of clones growing in the culture chamber. These growth curves could then be used to check the chosen values for the distributions of the dividing and newly formed organisms and also to gain more accurate information about the behaviour of organisms close to the points of formation and division. 


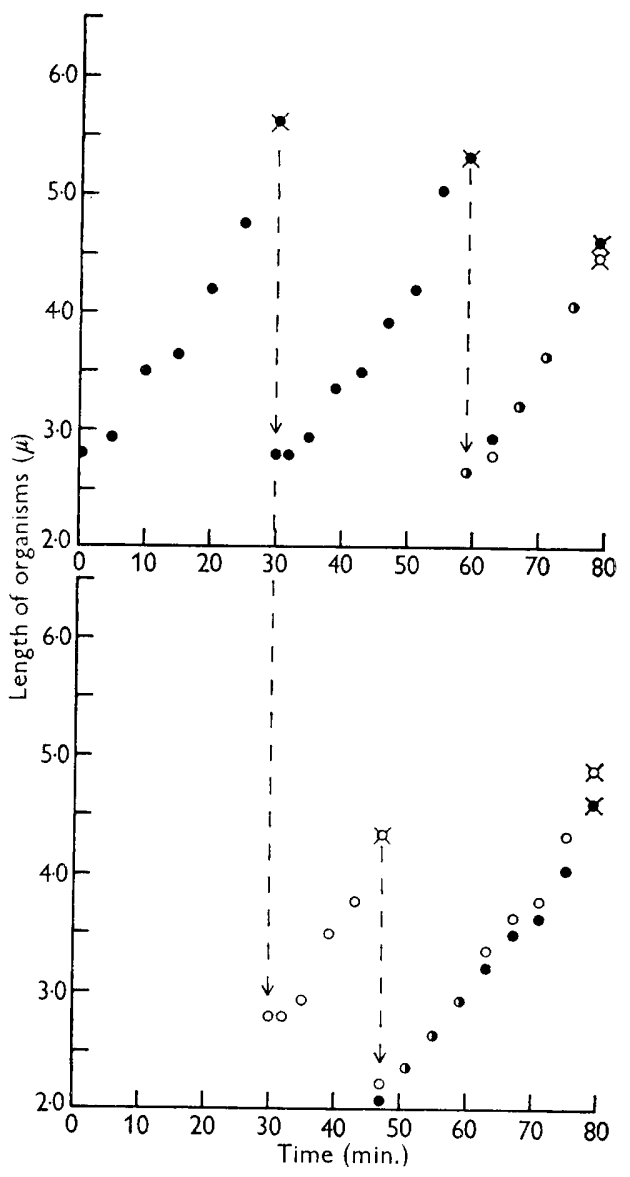

c

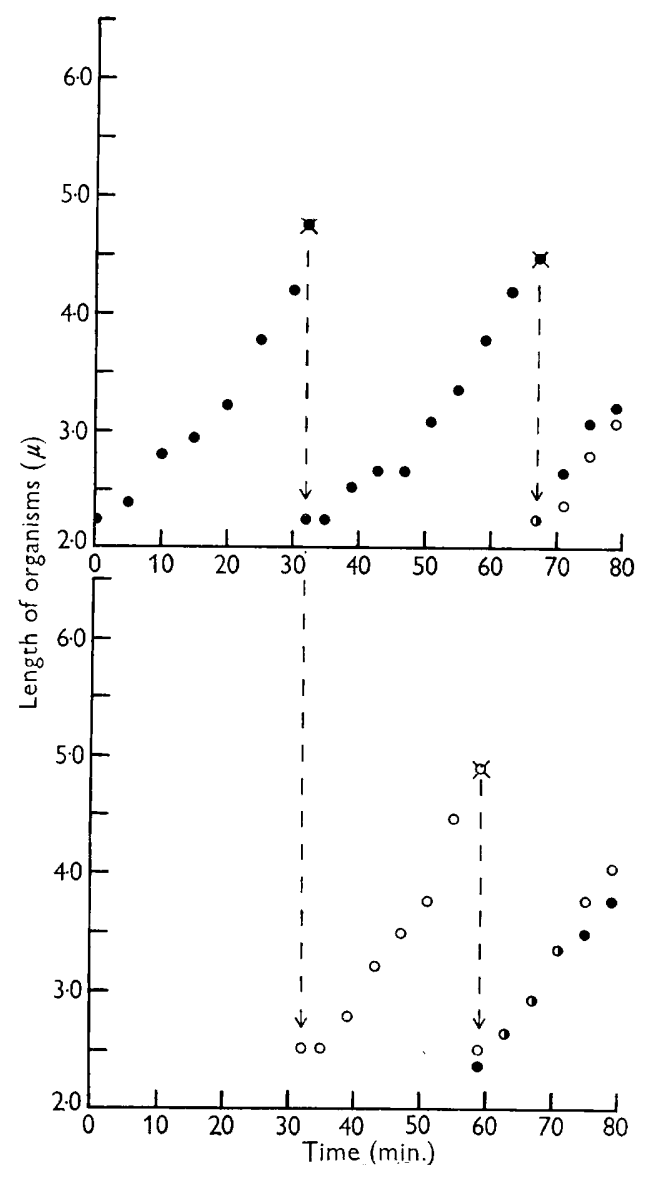

$d$

Fig. 4 (cont.). For legend see opposite.

For a valid comparison of the growth curves, the total rate of increase in length for all the organisms observed must be exponential and with a doubling time close to that of the bulk culture. A bulk culture was grown in $\mathbf{C H}$ medium until the growth had been exponential for several generations, and a few organisms were transferred to the culture chamber. The growth of these organisms was followed for at least two complete generations and the length of the organisms in the field was measured at 4 min. intervals. Nine clones were studied in this way, and the total length of the observed organisms was found to increase exponentially. Some typical growth curves are shown in Fig. 4. All the organisms showed an uninterrupted accelerating increase in length. These results, plotted in Fig. 5 in the same way as the results in Fig. 3, show that the results obtained with organisms in the culture chamber differ from the calculated results in Fig. 3 only at the extremes of the length range of the observed organisms, where the length distribution is most susceptible to error. In fact, the experimental curve is indistinguishable from any of the calculated curves over the range of lengths between $2 \cdot 5$ and $4.5 \mu$; this is the range in which most of the organisms spend the major part of the growth cycle. 
It was possible from the observed values of dividing and newly formed organisms in the culture chamber to obtain normal distributions (given in Table 2) which could be used with the length distribution from the bulk culture to calculate the rate of increase in length of organisms in the same manner as before. The result is shown in Fig. 6; the dashed line representing the observed growth rate as shown in Fig. 5. The agreement is good, hearing in mind that only 39 divisions were available

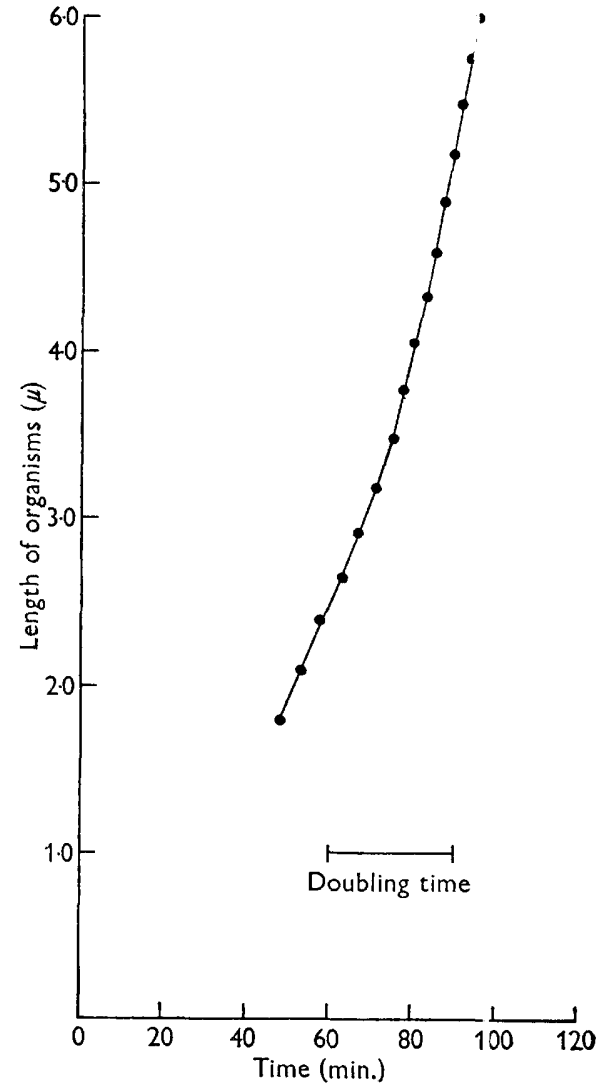

Fig. 5

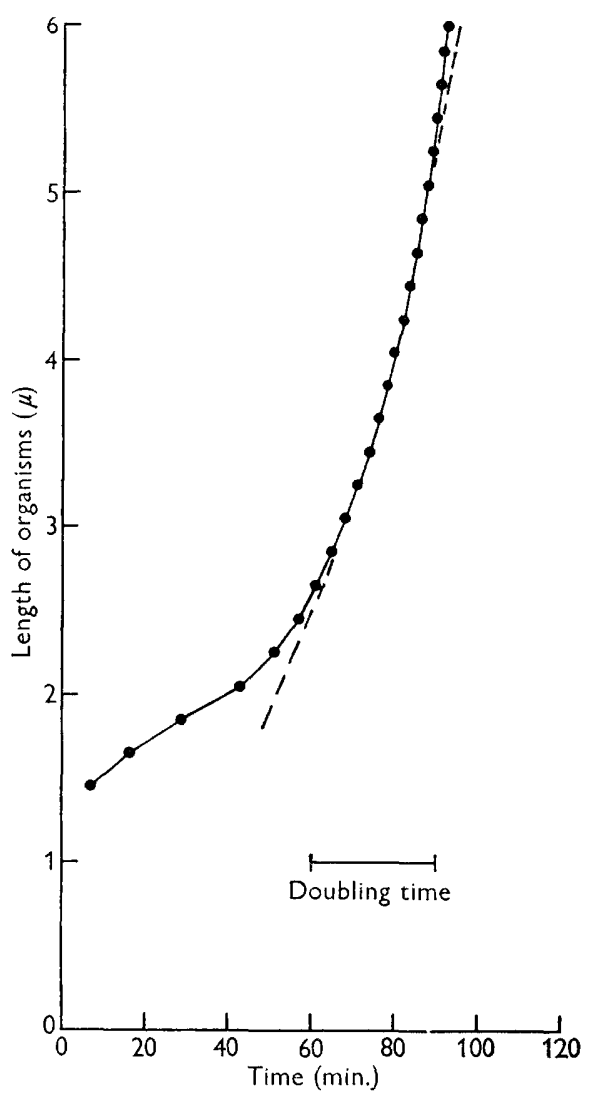

Fig. 6

Fig. 5. The rate of increase in length of Bacillus cereus between divisions based on all measurements on clones growing in the culture chamber. Doubling time $=30 \mathrm{~min}$.

Fig. 6. The rate of increase in leigth of Bacillus cereus between divisions based on the overall length distribution and the distributions of dividing and newly formed organisms observed in the culture chamber.

to produce the distributions us:ed, and that the observations were made every 4 min. This means that division could have occurred up to 4 min. earlier, when the organism would have been mark edly smaller than the length observed. This factor suggests that the parameters of the observed distributions are wrong; the mean lengths and the variances are too large, and a positive skewness will occur since the larger organisms increase in length faster than the smaller ones. 


\section{DISCUSSION}

The experiments described above show that it is possible to determine the rate of increase in length of bacteria between divisions by measuring the overall distribution of bacterial length from samples containing about $10^{3}$ bacteria taken from a bulk culture of organisms growing exponentially. The estimates of the distributions of the dividing and newly formed organisms which are required for the calculations can be made quite easily from the overall distribution of sizes of the organisms and do not necessarily involve experiments on single organisms in a culture chamber. Under the conditions used to grow Bacillus cereus $569 / \mathrm{H}$ on casein hydrolysate medium in bulk culture, the increase in length of the bacteria between divisions is found to be continuously accelerating and quite clearly not linear. It is similar to an exponential curve; but the rate of increase in length is not proportional to the length of the organism (as it should be if the increase were exponential), even in the region of the curves (Fig. 3) where all the estimates of the rate of increase are congruent. Furthermore, it is not possible to detect any halt or temporary fall in the rate of increase in length such as are present in sigmoid curves. Mitchison has recently observed the growth of Schizosaccharomyces pombe (1958) and Streptococcus faecalis (1961) which both show such halts or falls in the rate of increase of volume and dry weight during growth between divisions. The results calculated from the length distribution of the bulk culture are confirmed and extended by the series of observations made on clones growing in the culture chamber. The degree of agreement between these results shown in Figs. 3 and 5 is quite remarkable, and it has been possible to show that the calculated results in Fig. 3 are most in error at the two extremes of the length range, and that the behaviour of the organisms growing in the culture chamber is very consistent; there is no suggestion of any decrease in the rate of growth just after formation or immediately before division. From the observations in the culture chamber it was possible to calculate distributions for the dividing and newly formed organisms, which were also applied to the distribution of bacterial length from the bulk culture and gave the results shown in Fig. 6. The agreement between these results and those from culture chamber observations is again close, particularly over the central section of the graph. This part of the curve over which all the calculated results agree is the part in which most of the organisms spend the major part of the growth cycle between divisions, and the conclusions drawn from the shape of the curve in this region are in this instance completely reliable. Since the two end regions of the length distribution will contain errors due to the inexact application of the distinction between large single organisms and pairs of organisms formed by division (when the septum may be overemphasized or obscured), the calculated results for these regions are of less significance than the central portion, which is, however, more than adequate for a description of the behaviour of the majority of the organisms. The distributions producing the growth curve closest to the observed behaviour of the organisms in the culture chamber were those calculated from the observations of divisions in the culture chamber, although these distributions are not exact. The assumed distributions were given too small a variance, and the means chosen were in general smaller than those found from the culture chamber experiment. The effects of changes in mean or variance can be observed in Fig. 2, and in the light of these results it is possible to 
anticipate more clearly the modifications which should be made to improve any assumed distributions used for similar purposes.

The measurements of the rate of increase in length from both methods of observation confirm the earlier work of Knaysi (1940) on the growth of Bacillus cereus. Other patterns of increase in length may, however, be expected if the growth conditions are altered in such a way that one phase of the bacterial growth cycle between divisions takes significantly more or less time than the others. The uninterrupted increase in length observed for $B$. cereus under the conditions used must reflect a balanced state where the time taken to duplicate the genetic material is similar to that required to double the amount of cytoplasm in the organism and to synthesize sufficient cell wall material to form two new organisms at the moment of division.

The conclusions drawn about the increase in length between divisions do not allow similar conclusions to be drawn about the increase in volume or dry weight between divisions. For example, in a model case where the length increases exponentially, but the volume only increases linearly, the diameter of the organisms will increase and decrease $3 \%$ in the course of the division cycle-such a variation is beyond the limits of detection by the methods used for these experiments.

The general method of determining the rate of increase in length between divisions can be applied to the rate of increase of any chemical component (such as ribonucleic acid or deoxyribonucleic acid) or of any parameter (such as dry weight or volume) which can be conveniently measured on samples taken from a bulk culture which is growing exponentially. Moreover, since cultures growing exponentially show such a constant composition with regard to the size of the organisms, the method can also be used to investigate the effect on bacterial growth of compounds (such as antibiotics or amino acid 'analogues') which are known to interfere with the growth and division of bacteria.

\section{APPENDIX}

(1) Stability of certain features of a culture during ideal exponential growth

The growth of an exponentially growing culture may be expressed by the equation

$$
n=n_{0} e^{k t}
$$

where $n$ is the number of organisms at time $t, n_{0}$ the number of organisms at time 0 , $k$ is the growth constant, and $t$ is time. The constant, $k$, is equal to $\log _{e} 2 / T$, where $T$ is the observed doubling time. This exponential growth rate is also shared by the mass of the culture, and the average mass of a single organism is constant during this phase of the growth of the culture. Further, properties such as the average length of the organisms are also constant during exponential growth. Consider the average length of the organisms. A progressive increase or decrease in the average length would ultimately lead to an absurd situation. However, an 'oscillation' of the mean length of the organisms of a culture must be considered as a possible alternative to a constant mean length. Two points may be mentioned regarding this alternative. First, such an 'oscillation' of the mean length must be periodic and the mean length must have an average value with deviations both above and below this value. It is theoretically possible to produce a single culture by combining 
cultures at all stages of the mean length cycle such that this single culture has a mean length independent of time- - that is, a constant mean length. Secondly, cultures which are artificially disturbed in some way such that the mean length does oscillate (as, for instance, during growth of a 'synchronized culture') lose most traces of this initial state during a few generations, and tend toward a statc indistinguishable from that of the normal culture. It is evident that if individual bacteria behave sufficiently independently for this to occur, the final state must be equivalent to that of a culture composed of a large number of samples from cultures whose organisms have mean lengths randomly distributed throughout the 'periodic' mean length cycle; that is, the organisms in the culture will now have a constant mean length. Hence the independence of individual bacteria during exponential growth ensures that a balanced state is ultimately reached where the organisms in the culture have a constant mean length, even though this state may be attained either more or less rapidly. This conclusion is not true for cultures in other phases of bacterial growth.

Similar considerations show that when the mean length of the organisms in an exponentially growing culture is constant, the proportion of the total bacterial population of any given length is also constant. This population has what will be called a stable length distribution. The number of organisms of any length is therefore increasing exponentially with the same growth constant $k$, as the culture itself.

(2) Calculation of the rate of increase in length between divisions from the stable length distribution and the length distributions of dividing and neroly formed organisms

A. The rate of increase in numbers of organisms, $d n / d t$, is given by differentiating equation (i):

$$
d n / d t=k n_{0} e^{k t}=k n .
$$

Since each dividing organism gives rise to two daughters, the net increase in numbers per division is one organism. The rate of increase in numbers $(k n)$ is equal to the rate at which organisms divide, and new organisms are formed at twice this rate, i.e. $2 k n$.

Let the frequency function of the lengths of organisms at division be $\phi(l)$, where $l$ is length, and where

$$
\int_{0}^{\infty} \phi(l) d l=\mathbf{1}
$$

Then the number of organisms of length $l$ dividing in time $d t$ is

$$
k n \phi(l) \text {. }
$$

Similarly, let the frequency function of the lengths of newly formed organisms be $\psi(l)$, where

$$
\int_{0}^{\infty} \psi(l) d l=1
$$

The number of organisms of length $l$ formed in time $d t$ is

$$
2 k n \psi(l) \text {. }
$$

Consider a steady-state length distribution (Fig. 7), where $\lambda(l)$ is the frequency function of length and

$$
\int_{0}^{\infty} \lambda(l) d l=1
$$


The rate of increase in length of organisms of length $l_{x}$ will be denoted by $V_{x}$, and can be calculated in the following manner. If $p$ organisms lie between $l_{x}$ and $\left(l_{x}+d l\right)$ in length, and if $q$ organisms grow to length $l_{x}$ in a small time $d t$, then the group of organisms of length $l_{x}-\left(l_{x}+d l\right)$ is replaced $q / p$ times in time $d t$. For this to occur, organisms of length $l_{x}$ must be able to increase in length by the amount $d l q / p$ times in time $d t$. That is

$$
V_{x}=q / p \cdot d l / d t
$$

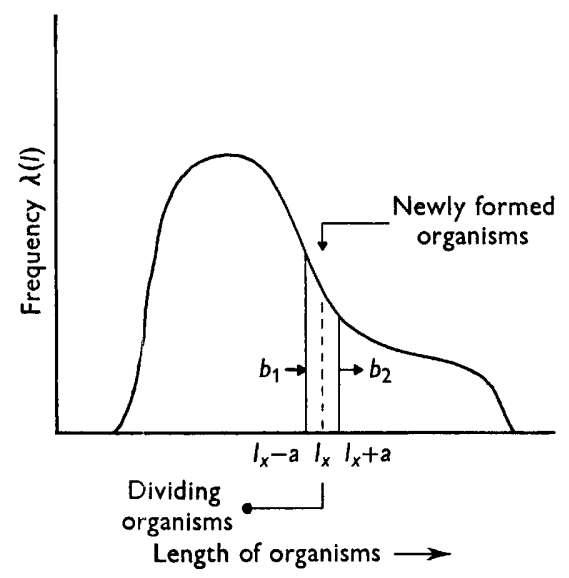

Fig. 7. A model length distribution of a stable population growing exponentially. The principal factors affecting the composition of any length category $\left(l_{x}-a\right)$ to $\left(l_{x}+a\right)$ are illustrated diagrammatically. The relationship between these rates is discussed in the text.

From the definition of $p$, it can be seen that

$$
p=n \lambda\left(l_{x}\right) d l \text {. }
$$

The number of organisms that reach $l_{x}$ in time $d t(q)$ equals the number of organisms formed with $l<l_{x}$ in time $d t$, minus the number of organisms which divide with $l<l_{x}$ in time $d t$, minus the number of organisms by which the population of organisms with $l<l_{x}$ increases in time $d t$ to maintain a constant proportion of the total population in this category as the total population increases. Hence

$$
q=2 k n d t \int_{0}^{l_{x}} \psi(l) d l-k n d t \int_{0}^{l_{x}} \phi(l) d l-k n d t \int_{0}^{l_{x}} \lambda(l) d l .
$$

Substituting for $p$ and $q$ in equation (v) we obtain

$$
V_{x}=k\left(2 \int_{0}^{l_{x}} \psi(l) d l-\int_{0}^{l_{x}} \phi(l) d l-\int_{0}^{l_{x}} \lambda(l) d l\right) / \lambda\left(l_{x}\right)
$$

The frequency distribution, $\lambda(l)$, and its integral are found from the measured length distribution, either from a fitted curve or approximated from the actual results, after they have been smoothed (e.g. by the method of Whittaker \& Robinson, 1948). If $l_{x}$ is chosen as the centre of one of the observed length groups, with limits $\left(l_{x}-a\right)$ to $\left(l_{x}+a\right)$ (see Fig. 7$)$, then the observed fraction of the population in this group is

$$
\int_{l_{x}-a}^{l_{x}+a} \lambda(l) d l, \text { and an approximation to } \lambda\left(l_{x}\right) \text { is } \int_{l_{x}-a}^{l_{x}+a} \lambda(l) d l / 2 a .
$$


Similarly, the integral

$$
\int_{0}^{l_{x}} \lambda(l) d l \text { may be approximated as } \int_{0}^{l_{x}-a} \lambda(l) d l+\frac{1}{2} \int_{l_{x}-a}^{l_{x}+a} \lambda(l) d l .
$$

$\int_{0}^{l_{x}-a} \lambda(l) d l$ is the fraction of the total population observed in all the length groups smaller than that with central value $l_{x}$. By this method $V_{x}$ can thus be readily calculated, and if it is assumed that the velocity at length $l_{x}$ is constant between $\left(l_{x}-a\right)$ and $\left(l_{x}+a\right)$, a continuous curve for the average growth of an organism may be obtained.

B. A modification of the above method of calculating $V_{x}$ can be made to calculate the average velocity of the organisms between the two limits of any observed length category. Let the limits of the length category be $\left(l_{x}-a\right)$ and $\left(l_{x}+a\right)$. If the average velocity of the organisms is $v$, then the organisms take time $2 a / v$ to cross the length category. Consider the organisms entering the length group at $\left(l_{x}-a\right)$ in time $d t$ when $t=0$ and when the total population is $n_{0}$. This batch $\left(b_{1}\right)$ is given by $q$ from equation (vii):

$$
b_{1}=k n_{0}\left(2 \int_{0}^{l_{x}-a} \psi(l) d l-\int_{0}^{l_{x}-a} \phi(l) d l-\int_{0}^{l_{x}-a} \lambda(l) d l\right) d t .
$$

This batch of organisms growing at a constant rate will reach any length $l$, where

$$
\left(l_{x}-a\right) \leqslant l \leqslant\left(l_{x}+a\right) \text { at a time } \frac{l-\left(l_{x}-a\right)}{v} ;
$$

organisms of length $l$ will be dividing at a rate given by equation (iii) as

$$
k n_{0} \exp \left[\frac{k}{v}\left(l-\left(l_{x}-a\right)\right)\right] \phi(l),
$$

and organisms of this length will be formed at a rate given by equation (iv) as

$$
2 k n_{0} \exp \left[\frac{k}{v}\left(l-\left(l_{x}-a\right)\right)\right] \psi(l)
$$

Hence the number of organisms in the batch will now be given by the expression $b_{1}-k n_{0} d t \int_{l_{x}-a}^{l} \exp \left[\frac{k}{v}\left(l-\left(l_{x}-a\right)\right)\right] \phi(l) d l+2 k n_{0} d t \int_{l_{x}-a}^{l} \exp \left[\frac{k}{v}\left(l-\left(l_{x}-a\right)\right)\right] \psi(l) d l$.

When $l=l_{x}+a$, when $t=2 a / v$, this group of organisms must be the same as the group of organisms $\left(b_{2}\right)$ that reach the length $\left(l_{x}+a\right)$ at this time, as calculated from equation (vii):

$$
b_{2}=k n_{0} \exp (2 k a / v)\left(2 \int_{0}^{l_{x}+a} \psi(l) d l-\int_{0}^{l_{x}+a} \phi(l) d l-\int_{0}^{l_{x}+a} \lambda(l) d l\right) d t
$$

Substituting $l=\left(l_{x}+a\right)$ in expression $(\mathrm{x})$, which then equals $b_{2}$ :

$$
\begin{aligned}
b_{2}=b_{1}-k n_{0} d t \int_{l_{x}-a}^{l_{x}+a} \exp [ & \left.\frac{k}{v}\left(l-\left(l_{x}-a\right)\right)\right] \phi(l) d l \\
& +2 k n_{0} d t \int_{l_{x}-a}^{l_{x}+a} \exp \left[\frac{k}{v}\left(l-\left(l_{x}-a\right)\right)\right] \psi(l) d l .
\end{aligned}
$$

Simple trapezoidal approximations are then made:

$$
\begin{array}{r}
\int_{l_{x}-a}^{l_{x}+a} \exp \left[\frac{k}{v}\left(l-\left(l_{x}-a\right)\right)\right] \phi(l) d l=\frac{1}{a}\left[\frac{1}{2} \phi\left(l_{x}-a\right)+\exp (k a / v) \phi\left(l_{x}\right)\right. \\
\left.+\frac{1}{2} \exp (2 k a / v) \phi\left(l_{x}+a\right)\right] .
\end{array}
$$


Similarly:

$$
\begin{aligned}
\int_{l_{x}-a}^{l_{x}+a} \exp \left[\frac{k}{v}\left(l-\left(l_{x}-a\right)\right)\right] \psi(l) d l=\frac{1}{a}\left[\frac{1}{2} \psi\left(l_{x}-a\right)+\exp (k a / v) \psi\left(l_{x}\right)\right. & \left.+\frac{1}{2} \exp (2 k a / v) \psi\left(l_{x}+a\right)\right] . \quad \text { (xiv) }
\end{aligned}
$$

Putting these approximations into equation (xii), and substituting for $b_{2}$ (equation (xi)) and $b_{1}$ (equation (ix)), and cancelling out the common term $k n_{0} d t$

$$
\begin{gathered}
\exp (2 k a / v)\left(2 \int_{0}^{l_{x}+a} \psi(l) d l-\int_{0}^{l_{x}+a} \phi(l) d l-\int_{0}^{l_{x}+a} \lambda(l) d l\right) \\
=\left(2 \int_{0}^{l_{x}-a} \psi(l) d l-\int_{0}^{l_{x}-a} \phi(l) d l-\int_{0}^{l_{x}-a} \lambda(l) d l\right) \\
-\frac{1}{a}\left[\frac{1}{2} \phi\left(l_{x}-a\right)+\exp (k a / v) \phi\left(l_{x}\right)+\frac{1}{2} \exp (2 k a / v) \phi\left(l_{x}+a\right)\right] \\
+\frac{2}{a}\left[\frac{1}{2} \psi\left(l_{x}-a\right)+\exp (k a / v) \psi\left(l_{x}\right)+\frac{1}{2} \exp (2 k a / v) \psi\left(l_{x}+a\right)\right] . \quad(\mathrm{xv})
\end{gathered}
$$

This can be solved as a quadratic equation in $\exp (k a / v)$, and knowing $\exp (k a / v)$, $v$ can be deduced. Equation (xv) contains the coefficient

$$
2 \int_{0}^{l_{x}+a} \psi(l) d l-\int_{0}^{l_{x}+a} \phi(l) d l-\int_{0}^{l_{x}+a} \lambda(l) d l
$$

which is equivalent to the coefficient

$$
2 \int_{0}^{l_{x}-a} \psi(l) d l-\int_{0}^{l_{x}-a} \phi(l) d l-\int_{0}^{l_{x}-a} \lambda(l) d l
$$

needed for the calculation of $v$ for the next length category; in this way the calculation can be set out economically in a tabular fashion; equally the pair

$$
\frac{1}{a}\left[\psi\left(l_{x}+a\right)-\frac{1}{2} \phi\left(l_{x}+a\right)\right]
$$

bear a similar relation to the pair

$$
\frac{1}{a}\left[\psi\left(l_{x}-a\right)-\frac{1}{2} \phi\left(l_{x}-a\right)\right] .
$$

Equation (xv) also contains only integral forms of $\lambda(l)$, with the upper limit always on a boundary between length groups; a simple smoothing of the observed length distribution is normally sufficient to produce these values, and the method thus avoids the necessity of fitting any definite function for $\lambda(l)$. Certain observed distributions (Henrici, 1928) with a shoulder or with double peaks, are difficult to fit to any simple function for $\lambda(l)$.

We would like to acknowledge a number of stimulating discussions with $\mathrm{Dr}$ J. M. Mitchison in the course of this work. Dr E. O. Powell made valuable suggestions, particularly concerning the quicker method A, shown in the Appendix. Miss M. V. Mussett was very helpful over the statistical problems associated with population distributions, and together with Miss S. Findley worked through many tedious calculations. The photomicrographs were prepared by $\mathbf{M r}$ M. R. Young and $\mathbf{M r} \mathbf{F}$. Wanless. We would like to thank the various friends and relations who lent projectors and measured bacterial cells on divers occasions. 


\section{REFERENCES}

Adolph, E. F. \& Bayne-Jones, S. (1932). Growth in size of micro-organisms measured from motion pictures. II. Bacillus megatherium. J. cell. comp. Physiol. 1, 409.

Bayne-Jones, S. \& Adolph, E. F. (1932a). Growth in size of micro-organisms measured from motion pictures. I. Yeast, Saccharomyces cerevisiae. J. cell. comp. Physiol. 1, 389.

BAYNE-JoNks, S. \& Adolph, E. F. (1932b). Growth in size of micro-organisms measured from motion pictures. III. Bacterium coli. J. cell. comp. Physiol. 2, 329.

Henrici, A. T. (1928). Morphologic Variation and the Rate of Growth of Bacteria. London: Baillière, 'Tindall and Cox.

Kantorowicz, O. (1951). Shaking apparatus for the aeration of bacterial cultures. J. gen. Microbiol. 5, 276.

KNaysi, G. (1940). A photomicrographic study of the rate of growth of some yeasts and bacteria. J. Bact. 40, 247.

KNaysi, G. (1941). A morphological study of Streptococcus faecalis. J. Bact. 42, 575.

Kogut, M., Pollock, M. R. \& 'Tridgell, F. J. (1956). Purification of penicillin-induced penicillinase of Bacillus cereus NRRI 569: a comparison of its properties with those of a similarly purified penicillinase produced spontaneously by a constitutive mutant strain. Biochem. J. 62, 391.

Mitchison, J. M. (1958). The growth of single cells. II. Saccharomyces cerevisiae. Exp. Cell. Res. 15, 214.

Mitchison, J. M. (1961). The growth of single cells. III. Streptococcus faecalis. Exp. Cell. Res. 22, 208.

Powell, E. O. (1955). Some features of the generation time of individual bacteria. Biometrika, 42, 16.

Powell, E. O. (1956). An improved culture chamber for the study of living bacteria. J. roy. micr. Soc. $75,235$.

Powell, IE. O. (1958). An outline of the pattern of bacterial generation times. J. gen. Microbiol. 18, 382.

Scherbaum, O. (1956). Cell growth in normal and synchronously dividing mass cultures of Tetrahymena pyriformis. Exp. Cell. Res. 11, 464.

Scherbaum, O. \& Rasch, G. (1957). Cell size distribution and single cell growth in Tetrahymena pyriformis GL. Acta path. microbiol. scand. 41, 161.

Ward, H. M. (1895). On the biology of Bacillus ramosus (Fraenkel), a schizomycete of the River 'Thames. Proc. Roy. Soc. 58, 265.

Whittaker, E. \& Robinson, G. (1948). The Calculus of Observations, 4th ed. p. $30: 3$. London: Blackie and Sons, Ltd. 